\title{
A field-deployable method for single and multiplex detection of DNA or RNA from pathogens using Cas12 and Cas13
}

\author{
Lina $\mathrm{Li}^{1}$, Canxing Duan ${ }^{1}$, Jianfeng Weng ${ }^{1}$, Xiantao $\mathrm{Qi}^{1}$, Changlin Liu ${ }^{1}$, Xinhai $\mathrm{Li}^{1,2}$, \\ Jinjie $\mathrm{Zhu}^{1^{*}}$ \& Chuanxiao Xie ${ }^{1^{*}}$ \\ ${ }^{1}$ National Key Facility for Crop Gene Resources and Genetic Improvement, Institute of Crop Science, Chinese Academy of Agricultural \\ Sciences (CAAS), Beijing 100081 China; \\ ${ }^{2}$ Biotechnology Research Institute, Chinese Academy of Agricultural Sciences (CAAS), Beijing 100081, China
}

Received August 31, 2021; accepted October 28, 2021; published online December 23, 2021

\begin{abstract}
For some Cas nucleases, trans-cleavage activity triggered by CRISPR/Cas-mediated cis-cleavage upon target nucleic acid recognition has been explored for diagnostic detection. Portable single and multiplex nucleic acid-based detection is needed for crop pathogen management in agriculture. Here, we harnessed and characterized RfxCas $13 \mathrm{~d}$ as an additional CRISPR/Cas nucleic acid detection tool. We systematically characterized AsCas12a, LbCas12a, LwaCas13a, and RfxCas13d combined with isothermal amplification to develop a CRISPR/Cas nucleic acid-based tool for single or multiplex pathogen detection. Our data indicated that sufficient detection sensitivity was achieved with just a few copies of DNA/RNA targets as input. Using this tool, we successfully detected DNA from Fusarium graminearum and Fusarium verticillioides and RNA from rice black-streaked dwarf virus in crude extracts prepared in the field. Our method, from sample preparation to result readout, could be rapidly and easily deployed in the field. This system could be extended to other crop pathogens, including those that currently lack a detection method and have metabolite profiles that make detection challenging. This nucleic acid detection system could also be used for single-nucleotide polymorphism genotyping, transgene detection, and qualitative detection of gene expression in the field.
\end{abstract}

nucleic acid detection, AsCas12a, LbCas12a, LwaCas13a, RfxCas13d, maize ear rot, Fusarium head blight, rice blackstreaked dwarf virus (RBSDV)

Citation: Li, L., Duan, C., Weng, J., Qi, X., Liu, C., Li, X., Zhu, J., and Xie, C. (2022). A field-deployable method for single and multiplex detection of DNA or RNA from pathogens using Cas12 and Cas13. Sci China Life Sci 65, 1456-1465. https://doi.org/10.1007/s11427-021-2028-x

\section{INTRODUCTION}

Pathogens such as bacteria, fungi, and viruses pose major threats to crop production and quality worldwide. For instance, the fungal pathogen Fusarium graminearum (FG) is the main cause of Fusarium head blight, a global, devastating disease of wheat (Triticum aestivum) and barley (Hordeum vulgare) (Bai and Shaner, 2004). FG also causes Gibberella ear rot and stalk rots in maize (Zea mays), resulting in yield

*Corresponding authors (Jinjie Zhu, email: zhujinjie@caas.cn; Chuanxiao Xie, email: xiechuanxiao@caas.cn) losses and poor product quality due to the presence of the trichothecene mycotoxins in grains (Wen et al., 2020; Yang et al., 2010). Fusarium verticillioides (FV), another multiphytopathogenic Fusarium species that is widely distributed throughout the world, threatens cereal production and food quality, producing toxic compounds such as fumonisins in maize and wheat grains (Deepa and Sreenivasa, 2017). FG and FV are closely related and can co-occur, resulting in maize root rot, stalk rot, and ear rot disease (Chandra Nayaka et al., 2009; Wen et al., 2020; Yang et al., 2010). In addition to fungal pathogens, the RNA virus rice black-streaked dwarf 
virus (RBSDV), a member of the genus Fijivirus in the family Reoviridae, naturally infects graminaceous crops including rice, wheat, and maize, leading to great yield losses (Cheng et al., 2013; Zhou et al., 2016). RBSDV viral RNAs are mainly transmitted by small brown planthopper (SBPH) in a propagative, persistent, and migratory manner among the rice, wheat, and maize hosts, leading to the rice blackstreaked dwarf, wheat dwarf, and maize rough dwarf diseases, respectively (Cheng et al., 2013).

The establishment of detection and monitoring methods for these pathogens and their intermediate hosts will contribute to crop protection and, therefore, crop production. Several direct or indirect methods have been developed to detect and monitor pathogens, including polymerase chain reaction (PCR), fluorescence in situ hybridization, immunofluorescence, flow cytometry, and gas chromatography-mass spectrometry (GC-MS) (Fang and Ramasamy, 2015). The specific nucleic acid features of pathogens (due to their genome diversity) form the basis for the development of direct, sensitive detection methods, even between closely related pathogens (Nimse et al., 2016). Nevertheless, it is still challenging to establish a field-deployable method that enables rapid identification of single and/or multiple closely related pathogens.

A number of bacterial systems based on clustered regularly interspaced short palindromic repeats (CRISPR) and CRISPR-associated protein (Cas) with RNA-guided RNAor DNA-targeting ability have been harnessed as sensitive nucleic acid detection tools (Abudayyeh et al., 2019; Chen et al., 2018; East-Seletsky et al., 2016; Gootenberg et al., 2017; Gootenberg et al., 2018; Harrington et al., 2018). The core rationale is that crRNA/Cas exhibits cis-cleavage activity upon target nucleic acid recognition that can trigger transcleavage activity on non-target reporter molecules, allowing for fluorescence or lateral-flow readout for nucleic acid detection (Chen et al., 2018; Gootenberg et al., 2017). The RNA-guided DNA targeting of Cas12a can trigger DNase cleavage of single-stranded (ss) DNA reporters in trans, whereas ssRNA is not capable of triggering trans-cleavage and is not susceptible to degradation by Cas12a (Chen et al., 2018). The RNA-guided RNase activities of Cas13a (formerly called $\mathrm{C} 2 \mathrm{c} 2$ ) allow for the sensitive detection of cellular RNA transcripts (East-Seletsky et al., 2016). New CRISPR/Cas systems, such as Cas12b (Teng et al., 2019) and Cas13d (EsCas13d and RspCas13d) (Qiao et al., 2021), continue to be developed as nucleic acid detection or genotyping tools. Cas $13 \mathrm{~d}$ has a remarkably compact protein size, and one ortholog derived from Ruminococcus flavefaciens XPD3002 (RfxCas13d) has highly efficient, robust, specific RNA interference activity in both mammals and plants (Konermann et al., 2018; Kushawah et al., 2020; Mahas et al., 2019; Yan et al., 2018; Zhou et al., 2020). The collateral activity of RfxCas $13 \mathrm{~d}$ was recently developed as a diag- nostic tool for SARS-CoV-2 (Brogan et al., 2020).

A sensitive, multiplex, field-deployable method is needed for pathogen monitoring in crops to successfully diagnose complex diseases due to co-occurring pathogens in a timely manner. The detection sensitivity of CRISPR/Cas is substantially improved to attomolar sensitivity when coupled with isothermal recombinase polymerase amplification (RPA) for DNA targeting and reverse transcription RPA (RTRPA) for RNA targeting, enabling detection of both DNA and RNA using Cas13 or Cas12 (Gootenberg et al., 2017). These findings provide a basis for designing simple tools for single and/or multiplex pathogen detection. One concern is the preparation of suitable field samples that meet the requirements as substrates for these systems. To enable fielddeployable diagnostics for detecting Zika and dengue viruses, heated bodily fluids were utilized as samples, resulting in sensitive detection within $2 \mathrm{~h}$ (Myhrvold et al., 2018). These studies provide an excellent basis for developing portable single or multiplex detection systems for crop pathogens in the field. However, the use of such systems to detect major crop pathogens has not been tested.

In this study, we (i) harnessed and characterized $\mathrm{RfxCas} 13 \mathrm{~d}$ as a nucleic acid detection tool; (ii) compared the kinetics of AsCas12a, LbCas12a, LwaCas13a, and RfxCas13d to develop good multiplex combinations; and (iii) developed a rapid, field-deployable diagnostic tool using both Cas 12 and Cas 13 to detect the above-mentioned RNA or DNA crop pathogens. Our newly developed single and/or multiplex detection system successfully detected important pathogens in the field in only 30-60 min. This system could also be used for the detection of other pathogens and for qualitative genotyping in the field.

\section{RESULTS}

\section{Harnessing CRISPR-RfxCas13d for nucleic acid detec- tion}

RfxCas13d has high in vivo targeting cleavage activity with important potential applications (Zhou et al., 2020). Here, we tried to reprogram RfxCas13d for RNA nucleic acid detection. First, we expressed RfxCas13d along with AsCas12a, LbCas12a, and LwaCas13a and confirmed their quality (Figure S1 in Supporting Information). When designing a nucleic acid detection system, the use of a specific signaling reporter and the sensitivity, kinetics, and specificity of spacer crRNA/target-triggered cleavage activity are core parameters that must be characterized. To identify ssRNA homopolymer reporters for RfxCas13d, we tested FAM-labeled ssRNA reporters with four different RNA base compositions. Reactions using poly(rU) ssRNA reporters displayed high specificity upon crRNA/target recognition. Therefore, labeled poly(rU) ssRNA reporters could enable sensitive 
fluorescence readouts, meeting the criteria for detection using RfxCas13d (Figure 1A).

To detect direct repeat (DR) sequences of crRNA, we tested seven different DRs from all currently known Cas13d orthologs (Table S1 in Supporting Information) (Konermann et al., 2018). The crRNAs with RfxDR and UrDR sequences exhibited faster kinetics than the others (Figure 1B). This finding helped us develop a rapid detection system with a readout time of 20-30 min using RfxCas13d. To test the crRNA/target specificity of RfxCas13d for detection, we tested a series of positional single- and double-mismatch spacer crRNAs (SMS and DMS) in the core region of crRNA from site 6 to 25 (Figure 1C and D; Table S1 in Supporting Information). The cleavage activity was significantly sensitive to SMS at positions 11, 13, and 17 (Figure 1C). Almost no DMS crRNAs with an additional mismatch at SMS11 generated a good detection signal (Figure 1D). We also performed target/crRNA specificity tests in which the mismatches were placed into the targets (Figure 1E). Analysis of the detection of sequences with mismatches between the crRNA/target in either direction (Figure 1C-E) indicated that the reprogrammed $\mathrm{RfxCas} 13 \mathrm{~d}$ successfully discriminated the targets with just 1-2-nucleotide specificity. Together, these results demonstrate that RfxCas13d could be used as a sensitive and specific nucleic acid detection tool.

\section{Kinetics of the detection sensitivity of RPA using Cas12 and Cas13 combined with DNA and RNA inputs}

AsCas $12 \mathrm{a}$ and LbCas12a preferentially cleaved polymer reporters at polyA, polyT, and polyC sites (Figure S2 in Supporting Information), whereas LwaCas13a and RfxCas 13d preferentially cleaved polyrU sites (Figure 1A; Figure S2 in Supporting Information). In addition, NEB CutSmart buffer showed the best compatibility with different Cas effectors with the best cleavage kinetics (Figure S3A in Supporting Information). These data lay the foundation for multiplex detection using Cas12 and Cas13 in the same reaction. Previous studies indicated that RPA for DNA targets and RT-RPA for RNA targets can substantially improve detection sensitivity and data readouts (Figure $2 \mathrm{~A}$ ). We therefore reasoned that the systematic evaluation of CRISPR/Cas systems would help us design a system for multiplex detection by excluding cleavage activity bias among enzymes. We conducted kinetic analysis of cleavage efficiency combined with RPA or RT-RPA upon serial dilution of synthetic DNA and RNA substrates (Figure 2B and C). A T7 promoter sequence was added to the $5^{\prime}$ end of the synthetic RPA primers and RT-RPA primers so that the RPA- or RT-RPAamplified DNA could be transcribed into ssRNA by T7 RNA polymerase. These T7-transcribed RNAs provided ssRNA

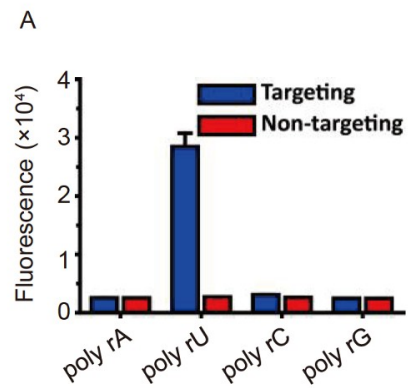

$$
\text { C }
$$

Single-Mismatch Spacer crRNA (SMS) SMS6 gGGCTTGTTCGTGGTTGAGA SMS7 CCGCTTGTTCGTGGTTGAGA .....

SMS24 CGGCTTGTTCGTGGTTGACA SMS25 CGGCTTGTTCGTGGTTGAGt PMS $\square \square$

Perfect Match Spacer (PMS) crRNA CGGCTTGTTCGTGGTTGAGA Fluor. $\left(\times 10^{4}\right) \frac{}{123}$

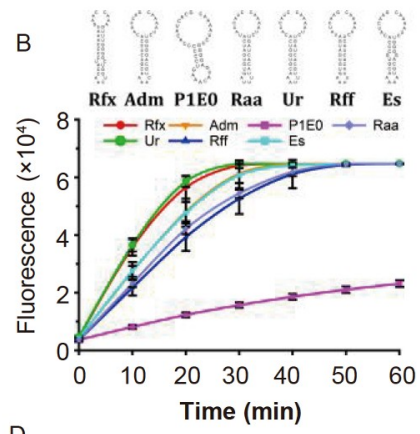

D

Double-Mismatch Spacer crRNA (DMS) DMS6 gGGCTaGTTCGTGGTTGAGA

DMS7 CcGCTaGTTCGTGGTTGAGA .....

DMS24 CGGCTaGTTCGTGGTTGACA DMS25 CGGCTaGTTCGTGGTTGAGt

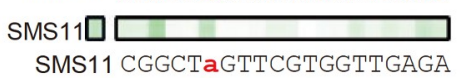

Fluor. $\left(\times 10^{4}\right)$

123

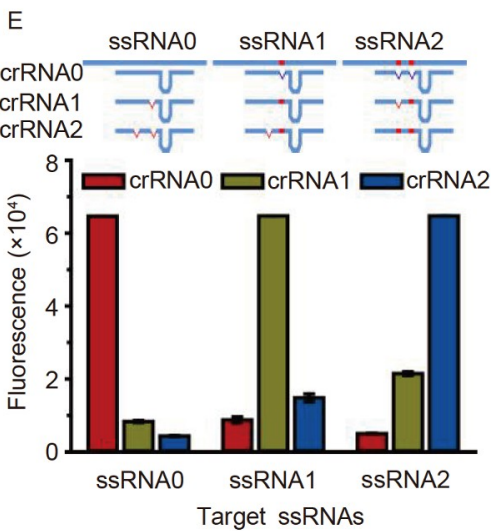

Figure 1 Harnessing and characterizing RfxCas13d as a nucleic acid detection tool. A, Identification of an ssRNA homopolymer reporter that could be used with RfxCas13d. B, Characterization of the targeting efficiency of RfxCas13d for different DRs from seven reported Cas13d orthologs (Konermann et al., 2018). C and D, crRNA/target specificity test using mismatches in crRNA. The targeting efficacy of RfxCas13d for crRNAs with a single mismatch (SMS) and double mismatch (DMS) at serial positions in the core spacer RNA region from site 6 to 25 . The fluorescence intensities are shown corresponding to the SM and DM positions. Position 11 was selected as the fixed site of DM based on the results of SM sensitivity tests. Perfect match spacer crRNA (PMS) was used as a control. E, Target/crRNA specificity test using mismatches in targets. Collateral activity of RfxCas13d on ssRNA0 (original ssRNA), ssRNA1 (original ssRNA with a single mutation at position 11), and ssRNA3 (original ssRNA with a double mutation at positions 11 and 13) with crRNA0 (original crRNA), crRNA1 (original crRNA with a single mutation at position 11), and crRNA2 (original crRNA with a double mutation at positions 11 and 13). The red bumps in the crRNAs indicate the mismatch sites. The red dots in the crRNA1, crRNA2, and ssRNA1, ssRNA2 indicate the positions with different nucleotides in crRNA0 and ssRNA0, respectively. All data were produced from three technical replicates, and data are shown as mean \pm SD. 
targets for LwaCas13a and RfxCas13d, resulting in collateral effects on crRNA/Cas13a target recognition.

As observed in the images in Figure 2B and $\mathrm{C}$ above each panel, $10^{9}$ attomolar (aM; equal to $10^{-9} \mathrm{~mol} \mathrm{~L}^{-1}$ ) of DNA or RNA input could easily be distinguished using Cas 12 with non-RPA (Figure 2B, two left panels) and using Cas 13 with non-RT-RPA (Figure 2C, two right panels). Combined with RPA or RT-RPA, the detection sensitivity of the Cas 12 and Cas 13 systems was approximately $1 \mathrm{aM}$ (Figure 2B and C), which allowed us to detect a $20-\mu \mathrm{L}$ reaction containing only a few copies of target DNA/RNA molecules. These results indicate that the Cas12- and Cas13-based systems have sensitive detection capacities.

\section{Single and/or multiplex detection of the DNA pathogens FG and FV}

FG and FV are two major pathogens that cause maize ear rot disease, resulting in yield losses and poor crop quality (Figure 3A). Preliminary verification experiments based on PCR detection confirmed the identities of FG and FV (Figure
S4 in Supporting Information). We then tested the single and/ or multiplex detection systems using samples from the field. To discriminate between these two fungi, we obtained crude DNA extracts by subjecting the samples to lysis for $6 \mathrm{~min}$ at $37^{\circ} \mathrm{C}$ and used them as substrates for direct detection via RPA. Both the fluorescent signals and the data readout indicated that all four Cas effectors successfully distinguished between FV (Figure 3B) and FG (Figure 3C) using a single target site. These pathogens were also detected qualitatively using LbCas12a-based lateral-flow strips (Figure 3D).

DNA and/or RNA homopolymers with different labels are ideal materials for multiplexing between Cas 12 and Cas 13 due to their different base preferences for collateral nuclease activity. We chose LbCas12a and LwaCas13a to target two different ssDNA and/or ssRNA sites, customized ssDNA reporters for LbCas12a were labeled with HEX fluorescein, and ssRNA reporters for LwaCas13a were labeled with FAM fluorescein. Then, we successfully performed multiplex detection of double targets of FV (Figure 3E) and FG (Figure $3 \mathrm{~F})$ by combining LbCas12a and LwaCas13a. We also attempted to detect both FV and FG in a single reaction by
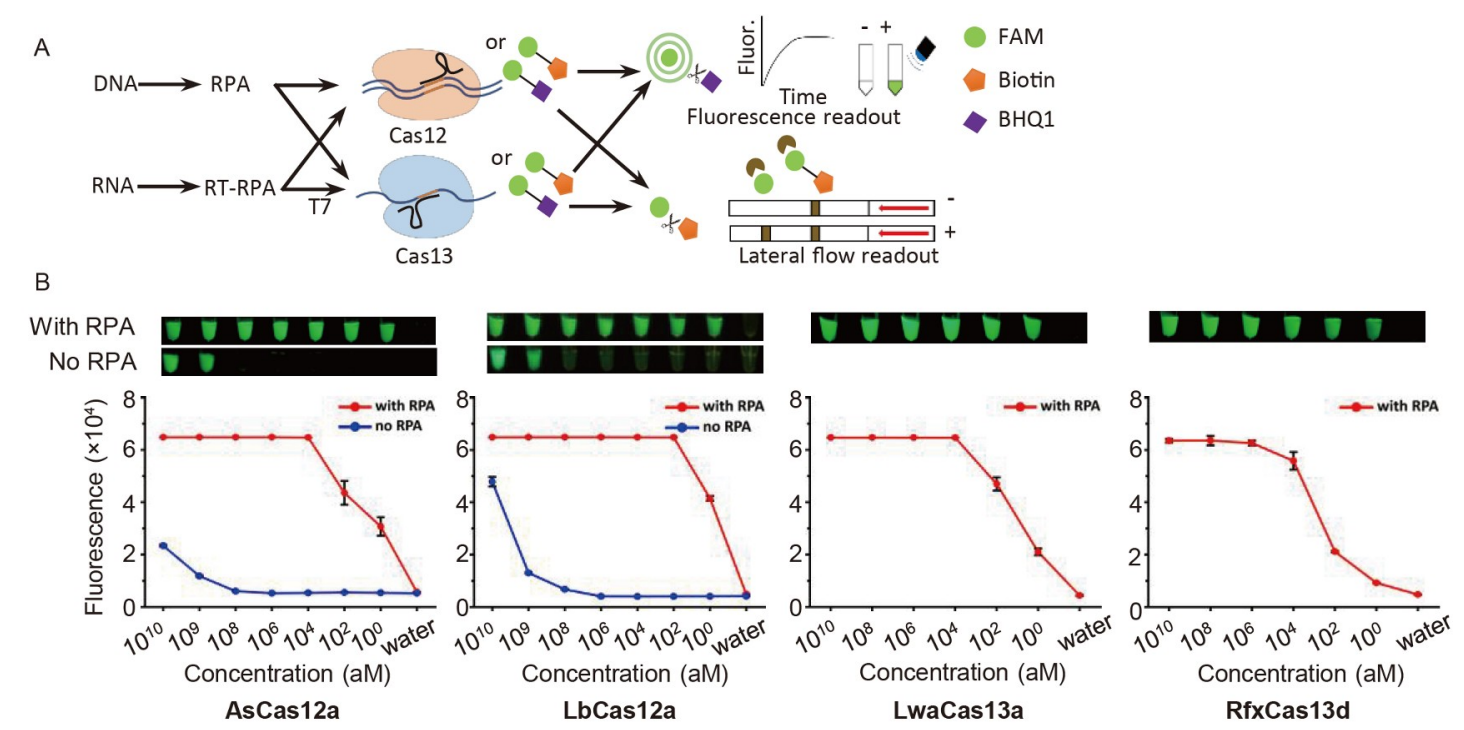

C
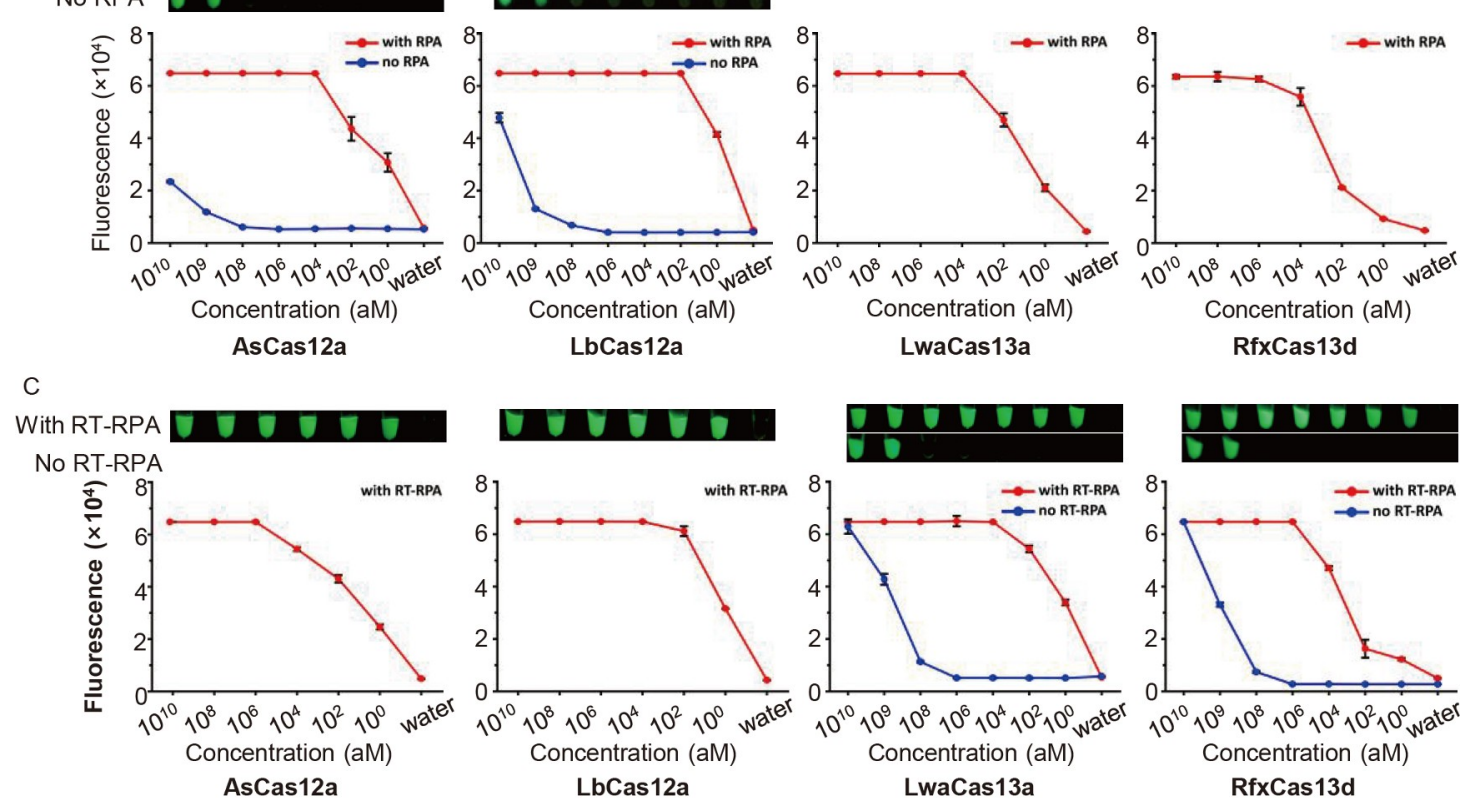

Figure 2 Kinetics of detection sensitivity using Cas12 and Cas 13 with DNA and RNA inputs. A, Diagram of nucleic acid detection using Cas 12 or Cas13 to detect DNA and RNA. B and C, The detection sensitivity of four nucleic acid detection systems using different concentrations of DNA (B) and RNA (C) as target inputs. Synthetic DNA (B) or RNA (C) substrate was diluted to different concentrations and added to the CRISPR/Cas12a or CRISPR/Cas13 reaction with or without RPA or RT-RPA amplification. Fluorescent signals were monitored, and photographs taken at $30 \mathrm{~min}$ (for CRISPR/Cas12) or $60 \mathrm{~min}$ (for CRISPR/Cas13). $n=3$ technical replicates; data are shown as mean \pm SD. 
mixing the positive samples as input (Figure 3G). Both the visible fluorescent signals and data readout indicated that we successfully determined whether maize exhibiting ear rot was infected with FG and/or FV using only a single test. We also collected data on the detection sensitivity using this crude DNA extract as the substrate. The assay was sensitive up to a dilution of $10^{5}$-fold (Figure S5 in Supporting Information).

\section{Detection of the RNA pathogen RBSDV}

We also tested the use of our field-deployable detection assay for RBSDV, an RNA viral pathogen that is transmitted by SBPH and infects rice, wheat, and maize. The presence of RBSDV in these four different hosts (SBPH, rice, wheat, and maize) was verified by RT-PCR (Figure S6 in Supporting Information). To enable detection in the field, we optimized the method for crude RNA extract preparation to meet the requirements for portable detection outside of the laboratory (MATERIALS AND METHODS). A lateral-flow readout using LbCas12a (Figure 4A) and visual fluorescence using both Cas 12 and Cas 13 with FAM-labeled probes (Figure 4BE) qualitatively identified RBSBV in the field in all four hosts. These results indicate that this detection system is highly compatible with different plant and animal species. We also analyzed the detection sensitivity of our system using this crude RNA extract as substrate. This assay was sensitive up to a dilution of $10^{4}-10^{5}$-fold (Figure S7 in Supporting Information).

\section{Minimal time and equipment are needed for portable single or multiplex pathogen detection}

Field-deployable systems are used to achieve real-time onsite testing. Such systems allow the detection and monitoring of crop pathogens to be enhanced for practical scenarios. Portable systems must be employed in a short period of time using minimal equipment. Using our system, the detection of a single DNA target required only $26 \mathrm{~min}$ per assay (Figure 5A). Multiplex detection took as long as $30 \mathrm{~min}$ since the kinetics of both Cas $13 \mathrm{~s}$ were slower than those of Cas $12 \mathrm{~s}$ (Figure 2B and C). At the 30-min time point, the LwaCas13a readouts were discernable but had slightly weaker signal strength than those of LbCas12a (Figure $3 \mathrm{E}-\mathrm{G}$ ). We also tested the ability to obtain a multiplex detection readout of fluorescence from the RNA virus RBSDV using LbCas12a
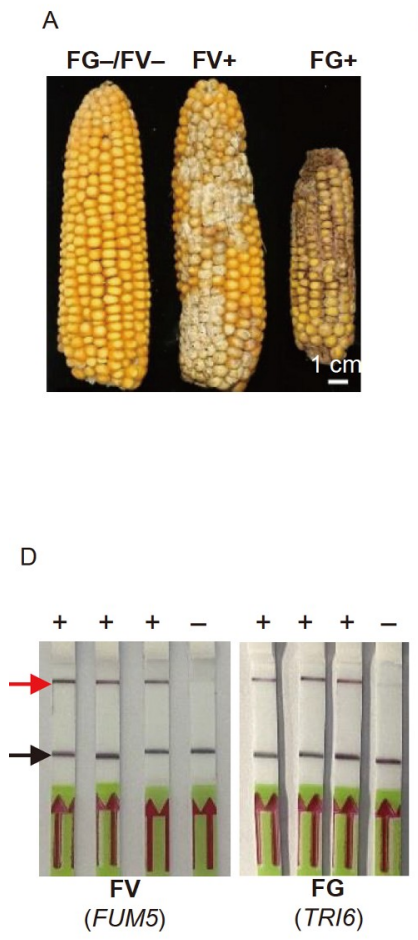
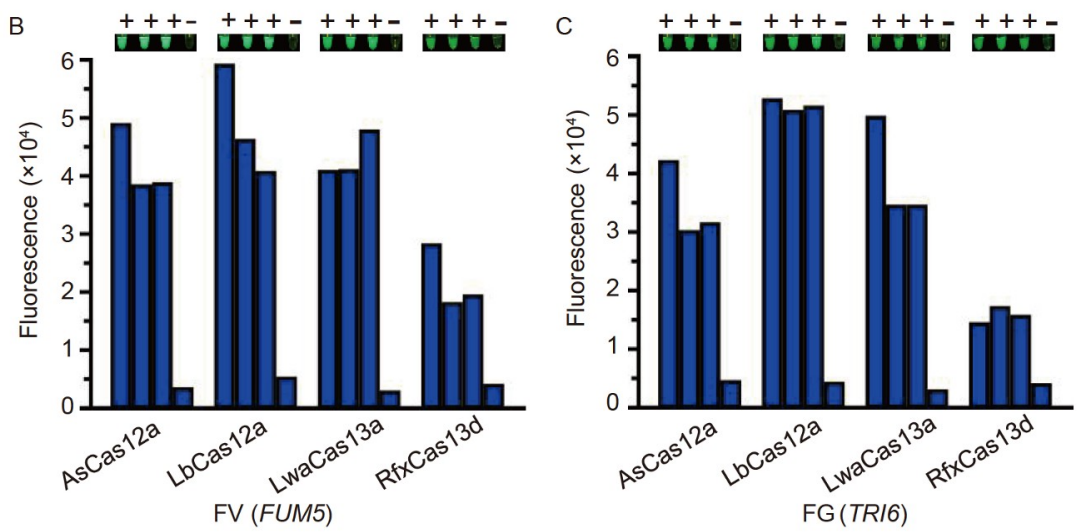

E

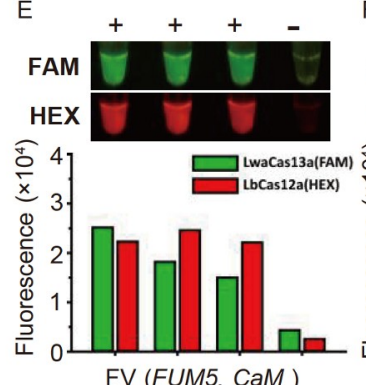

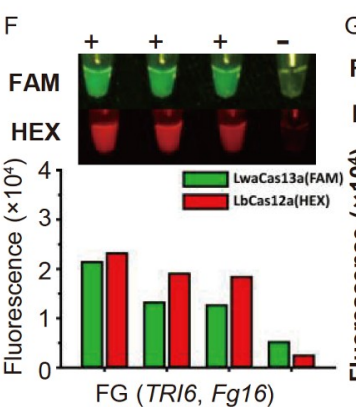

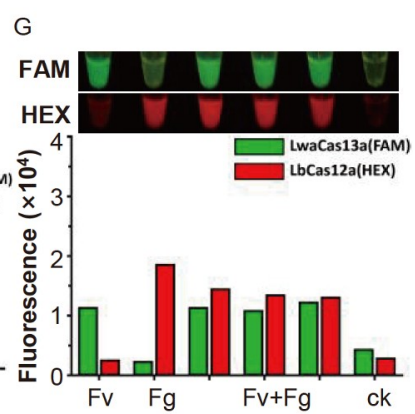

Figure 3 Single or multiplex detection of FG and FV on raw crude samples using Cas 12 and/or Cas 13. A, Maize ears infected with FG (FG+) or FV (FV+). B and C, Single detection of the DNA of fungal pathogens FV (FUM5 as the target) (B) and FG (TRI6 as the target) (C) using four CRISPR/Cas systems with a FAM fluorescent reporter. D, Fast lateral-flow readout detection of FV (left panel; FUM5 as the target) and FG (right panel, TRI6 as the target) using LbCas12a. E, Double-site multiplexed co-verification detection of FV (FUM5 and CaM as the double targets) using LbCas12a and LwaCas13a. F, Doublesite multiplexed co-verification detection of FG (TRI6 and Fg16 as the double targets) using LbCas12a and LwaCas13a. G, Multiplexed detection of FG (TRI6 as the target) and FV (FUM5 as the target) using LbCas12a and LwaCas13a (30 min).FUM5 and CaM, the specific targets used to identify FV; TRI6 and $F g 16$, the specific targets used to identify FG. +, RNA/DNA pathogen-borne sample; -, uninfected controls. Black arrowhead, quality control band; red arrowhead, positive band. 

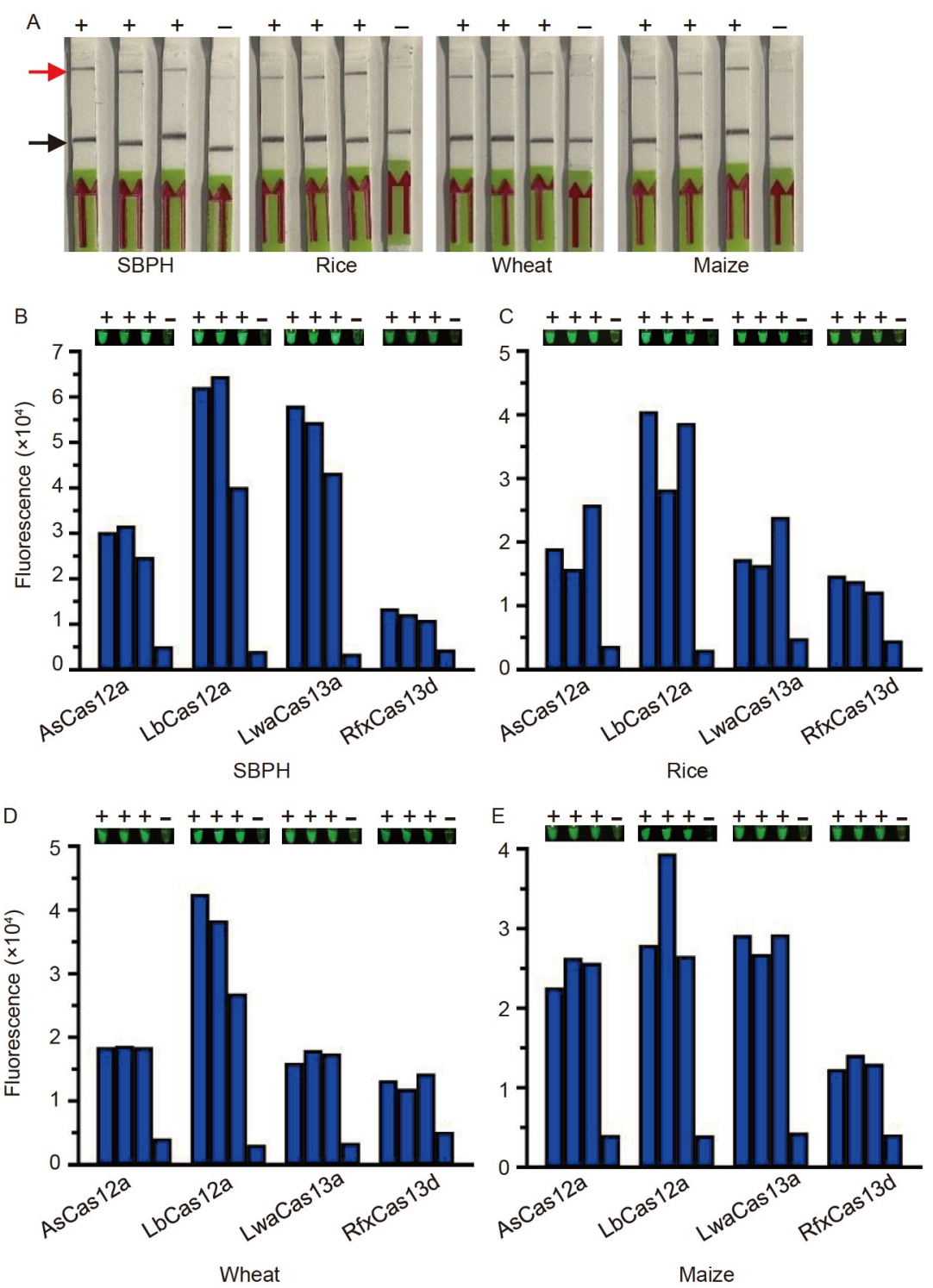

Figure 4 Detection of the RNA virus RBSDV in diverse hosts. A, Lateral-flow readout detection of RBSDV using LbCas12a. B-E, Fluorescent readout detection of the virus carried by SBPH (B) and infected rice (C), wheat (D), and maize (E) using the Cas12 and Cas13 systems. +, RNA/DNA pathogen-borne sample; -, uninfected controls. Black arrowhead, quality control band; red arrowhead, positive band.

and LwaCas13a in 30 min (Figure 5B). The visual and data readout indicated that this system functioned well, although some bias was found between LbCas12a and LwaCas13a. All of the equipment required, from sampling to reactions (Figure 5C) to visual readouts (Figure 5D), are shown in Figure 5. Our data indicate that our portable detection systems could be rapidly and easily deployed in the field.

\section{DISCUSSION}

\section{Cas12- and Cas13-based nucleic acid detection}

Previous reports (Kellner et al., 2019; Xiong et al., 2020) and the current data (Figures 2-4) consistently show that the CRISPR/Cas12a system has some advantages over Cas13 systems in terms of sensitivity and reaction speed. However, Cas13-based systems remain competitive for detection and diagnosis. For instance, a recently developed amplificationfree RNA-sensitive detection method was successfully developed using Cas13a and Csm6 in tandem (Liu et al., 2021), highlighting the advantages of Cas 13 family members as tools for nucleic acid diagnosis with a wide range of applications. The RNA-guided RNase Cas13d is approximately $20 \%$ smaller than Cas13a-Cas13c (Yan et al., 2018). Its compact structure and small size are beneficial for both in vivo and in vitro applications. For instance, CRISPR/Cas $13 \mathrm{~d}$ systems were successfully employed for a high-throughput study of functional circRNAs (Zhang et al., 2021), RNA virus interference (Mahas et al., 2019), and mRNA knockdown (Kushawah et al., 2020). 

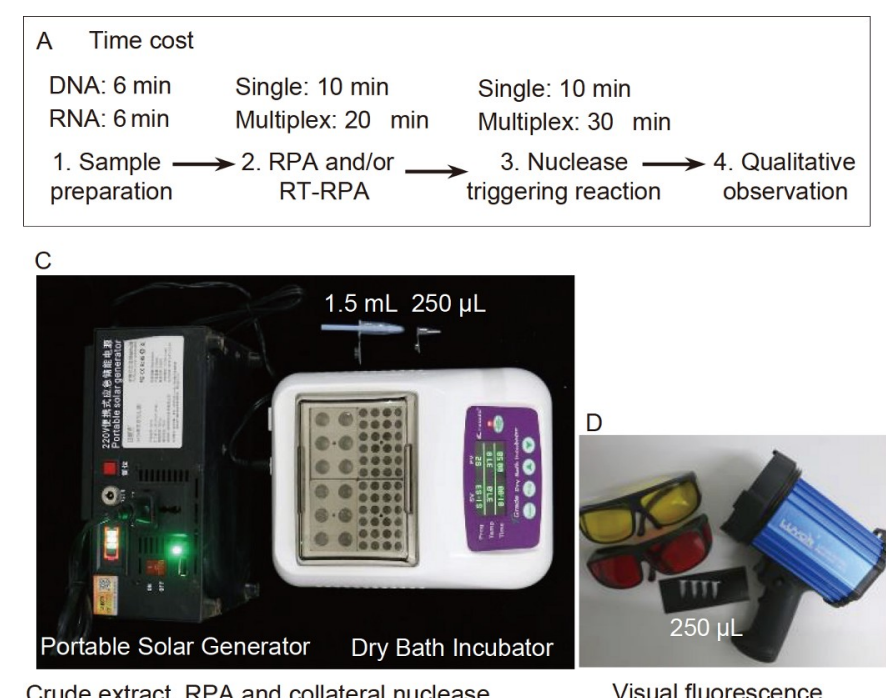

Crude extract, RPA and collateral nuclease

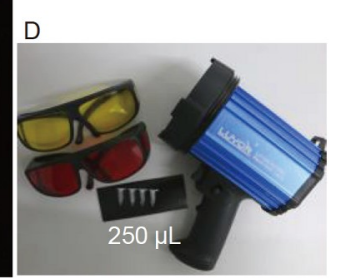

Visual fluorescence
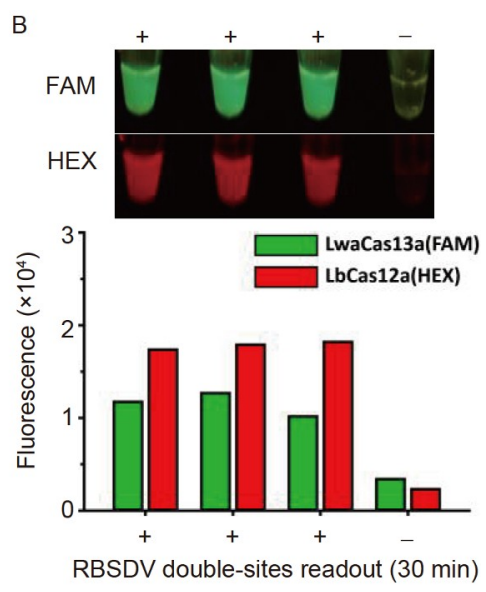

RBSDV double-sites readout (30 min)

Figure 5 Minimal time and instrument requirements for field-deployable multiplex monitoring through nucleic acid detection with Cas12 and Cas13. A, Time required for the procedures from sampling to readout. B, Multiplex detection readout of fluorescence of RBSDV via LbCas12a and LwaCas13a performed in $30 \mathrm{~min}$. C, Minimal portable instruments required in the field. A portable solar generator (Bei-Shi-Te ${ }^{\mathrm{TM}}$, Shenzhen, China) and dry bath incubator (OSE-DB-01, Tiangen Biotech (Beijing) Co., Ltd., China) were used to prepare crude extracts and perform the RPA and collateral nuclease reactions. Eppendorf tubes were used for crude extract preparation $(1.5-\mathrm{mL}$ tube) and reactions $(250-\mu \mathrm{L}$ tube). A small grinding rod was used to grind each sample in a 1.5-mL Eppendorf tube. D, A handheld LUYOR-3415RG instrument (LUYOR, USA) with a 488-nm excitation filter (yellow glasses) for the FAM reporter and a 520-nm excitation filter (red glasses) used for visual observation of the HEX reporter.

In this study, we reprogrammed the collateral cleavage activity of RfxCas13d for nucleic acid detection. The transcleavage preference profile of $\mathrm{RfxCas} 13 \mathrm{~d}$ was similar to that of LwaCas13a. Using RfxCas13d, we achieved attomolar detection sensitivity for both DNA and RNA targets. However, a slightly slower reaction speed was obtained using low target inputs compared with Cas13a. To help overcome this drawback, perhaps transcription loop-mediated isothermal amplification (RT-LAMP) (Ali et al., 2020) could be used to optimize the reaction speed.

These detection systems could also have applications beyond crop pathogen identification. For example, the collateral activity of CRISPR/Cas12 and CRISPR/Cas 13 systems has been leveraged for high-fidelity SNP genotyping (Teng et al., 2019) and clinical disease diagnosis, especially for detecting SARS-CoV-2 (severe acute respiratory syndrome coronavirus 2) (Jiao et al., 2021; Liu et al., 2021; Xiong et al., 2020).

\section{Sensitive field-deployable nucleic acid detection}

We determined that CRISPR/Cas-based nucleic acid detection could detect $10 \mathrm{nmol} \mathrm{L}^{-1}$ targets without pre-amplification of RPAs, while the detection sensitivity reached attomolar levels when coupled with pre-amplification (Figure $2 \mathrm{~B}$ and $\mathrm{C}$ ). For practical applications, we recommend screening for primer combinations with high amplification rates and specificity prior to detection. RPA or RT-RPA treatment can substantially improve the detection sensitivities of all CRISPR/Cas-based nucleic acid detection sys- tems. However, thermostatic amplification of RPA alone cannot achieve high sensitivity detection (Gootenberg et al., 2017). The collateral cleavage triggered by the crRNA/Cas effector upon target recognition is another important component of this technology. Therefore, CRISPR/Cas and RPA represent two core components for detection systems.

Here, to meet the requirements of portable detection in the field, we used a portable solar generator and a small dry bath incubator, which allowed us to generate crude extracts and perform RPA and nuclease trigger reactions in the field (Figure 5C). The fluorescent readout generated by multiplex detection in the same tube (Figure 5B) was based on the use of diverse reporters of LbCas12a/LwaCas13a, generating signals of different colors under different excitation wavelengths provided by a handheld light with filter glasses (Figure 5D). For lateral-flow readouts (Figures 3D and 4A), biotin-labeled ssDNA was used for the detection reaction, enabling device-free observation. Our results demonstrate the great potential of our system for the diagnosis, detection, and monitoring of crop pathogens in the field.

\section{MATERIALS AND METHODS}

\section{Protein expression and purification}

The AsCas12a, LbCas12a, LwaCas13a, and RfxCas13d genes were cloned into the linearized pET-30a $(+)$ expression vector. Cas proteins were purified as previously described (Rees et al., 2017) with slight modifications. Protein purification steps were conducted sequentially using Ni-NTA 
affinity, ion exchange, and size-exclusion columns at $4^{\circ} \mathrm{C}$. The purified Cas proteins were pooled, and the buffer was exchanged for storage buffer.

\section{Synthetic targets, reporters, crRNAs, and primers used in this study}

Synthetic DNA templates were generated by oligonucleotide extension using a KAPA HiFi PCR Kit (Kapa Biosystems, USA) and cleaned using PCR purification columns (QIAGEN, Germany). For RNA targets and crRNA, purified double-stranded DNA template was incubated with T7 polymerase overnight at $37^{\circ} \mathrm{C}$ using a HiScribe T7 Quick High Yield RNA Synthesis Kit (NEB, USA) and purified with an RNA Clean and Concentrator Kit (QIAGEN). All crRNA sequences used in this study are listed in Table S1 in Supporting Information.

The ssDNA and ssRNA reporters were custom synthesized by Synbio Technologies (Suzhou, China). The reporters used in this study are listed in Table S2 in Supporting Information. The detailed sequences of the synthetic DNA and RNA targets used in this study are shown in Table S3 in Supporting Information. Primers for PCR, RT-PCR, RPA, and RT-RPA were designed using NCBI Primer-BLAST, with the T7 promoter sequence added to the forward or reverse primer used for Cas13. The primers were custom synthesized (Sangon Biotech, Beijing, China). Information about the primers used in this study is provided in Table S4 in Supporting Information.

\section{Design of crRNAs for nucleic acid targeting}

The targets for the TRI6 and Fgl6 genes of FG (Bluhm et al., 2002; Zhou et al., 2018), FUM5 and CaM genes of FV (Bluhm et al., 2002; Mulè et al., 2004), and segments S5 and S10 of RBSDV (Wang et al., 2003; Zhou et al., 2017) were designed according to the protospacer adjacent motif (PAM) of each Cas effector (Abudayyeh et al., 2016; Zetsche et al., 2015). DNA oligos for in vitro transcription of crRNA guides were custom synthesized (Sangon Biotech).

\section{Preparation of crude DNA/RNA extracts for pathogen detection and verification}

Crude DNA extracts of FG and FV were obtained from 10-50 mg of three infected maize kernel tissues and one uninfected control. The kernels were collected, placed in 1.5-mL Eppendorf tubes, homogenized with a handheld tissue homogenizer in $100 \mu \mathrm{L}$ Lysis Buffer for Microorganisms (TaKaRa, Japan), and incubated at $37^{\circ} \mathrm{C}$ for $5 \mathrm{~min}$. The crude extracts were directly subjected to RPA and PCR. Crude RNA preparations were generated from three RBSDV-infected SBPH and rice, wheat, and maize leaves and one uninfected control at $14 \mathrm{~d}$ post infection. Briefly, $10-50 \mathrm{mg}$ of leaf tissue was collected, placed into a $1.5-\mathrm{mL}$ tube, homogenized with a handheld tissue homogenizer in $100 \mu \mathrm{L}$ RT-qPCR Sample Preparation Reagent (Bio-Rad, USA), and incubated at $37^{\circ} \mathrm{C}$ for $5 \mathrm{~min}$. To prepare crude extracts from SBPH, the insect was placed into a $250-\mu \mathrm{L}$ PCR tube, ground in $20 \mu \mathrm{L}$ RT-qPCR Sample Preparation Reagent (Bio-Rad), and incubated at $37^{\circ} \mathrm{C}$ for $5 \mathrm{~min}$.

PCR verification of FG and FV and RT-PCR verification of RBSDV were performed in the laboratory using crude DNA or RNA extracts as substrates. Routine PCR was conducted for FG and FV, while RT-PCR was performed with a HiScript One Step RT-PCR Kit (Vazyme, Nanjing, China) to verify the presence of RBSDV.

\section{RPA, RT-RPA, and nuclease trigger reactions in the field}

A small dry bath incubator (OSE-DB-01, Tiangen Biotech) with a portable solar generator (Bei-Shi-Te ${ }^{\mathrm{TM}}$ ) was used for the crude extract reaction, RPA, and nuclease trigger reactions in the field.

For RPA reactions, a TwistAmp Basic RT-RPA Kit (TwistDX, UK) was used according to the manufacturer's instructions; the sample was incubated at $37^{\circ} \mathrm{C}$ for $10 \mathrm{~min}$. For RT-RPA reactions, $0.5 \mu \mathrm{L}$ Reverse Transcriptase (NEB) was added to the RPA basic reaction, and the samples were incubated at $37^{\circ} \mathrm{C}$ for either $10 \mathrm{~min}$ (single target detection) or $30 \mathrm{~min}$ (sensitivity detection). For multiplex RPA or RT$\mathrm{RPA}$ reactions, the reaction was incubated at $37^{\circ} \mathrm{C}$ for $20 \mathrm{~min}$.

In general, $1 \mu \mathrm{L}$ original targets or RPA and RT-RPAamplified products was added to $19 \mu \mathrm{L}$ prepared CRISPR/ Cas reaction mixture containing $1 \times$ working buffer, $100 \mathrm{nmol} \mathrm{L}^{-1}$ purified Cas proteins, $0.5 \mu \mathrm{L}$ murine RNase inhibitor (NEB), $1 \mu \mathrm{L}$ transcribed crRNA $\left(20 \mathrm{ng} \mu \mathrm{L}^{-1}\right)$, and $2 \mu \mathrm{mol} \mathrm{L}^{-1}$ ssDNA (Synbio Technologies) or ssRNA reporter (Synbio Technologies). For Cas13a detection, the above reaction also included $0.05 \mu \mathrm{L}$ T7 RNA polymerase (NEB) and $0.8 \mu \mathrm{L}$ rNTP Mix (NEB). For multiplex detection assays, the reaction mixture contained $1 \times$ working buffer, $100 \mathrm{nmol} \mathrm{L}^{-1}$ purified Cas13a proteins, $100 \mathrm{nmol} \mathrm{L}^{-1}$ purified Cas 12 a proteins, $0.5 \mu \mathrm{L}$ murine RNase inhibitor (NEB), $1 \mu \mathrm{L}$ transcribed crRNA1 $\left(20 \mathrm{ng} \mu \mathrm{L}^{-1}\right), 1 \mu \mathrm{L}$ transcribed crRNA2 $\left(20 \mathrm{ng} \mu \mathrm{L}^{-1}\right), 0.05 \mu \mathrm{L}$ T7 RNA polymerase, $0.8 \mu \mathrm{L}$ rNTP Mix (NEB), $2 \mu \mathrm{mol} \mathrm{L}^{-1}$ ssDNA (Synbio Technologies), and $2 \mu \mathrm{mol} \mathrm{L}^{-1}$ ssRNA reporter (Synbio Technologies).

Fluorescence and lateral-flow readout assay in tubes and scoring fluorescence intensity data

For visual observation, CRISPR/Cas reactions were incubated at $37^{\circ} \mathrm{C}$ for $10-30 \mathrm{~min}$ and detected using a 
LUYOR-3415RG instrument (LUYOR, USA) with a 488$\mathrm{nm}$ excitation filter (for the FAM-quencher reporter) or 520$\mathrm{nm}$ excitation filter (for the HEX-quencher reporter). Multiplex observation was achieved by shifting the $488-\mathrm{nm}$ excitation filter used for the FAM-quencher reporter to a 520$\mathrm{nm}$ excitation filter used for the HEX-quencher reporter in the same reaction tubes. Lateral-flow detection was performed with commercial strips (TwistDX). Fluorophore quencher-labeled reporters were replaced by $1 \mu \mathrm{mol} \mathrm{L}{ }^{-1}$ FAM biotin-labeled ssDNA (Synbio Technologies). After a 10-min incubation, $80 \mu \mathrm{L}$ HybriDetect Buffer was added to the reaction, and the samples were run on the HybriDetect lateral-flow strips.

Fluorescence intensity data were scored in a real-time PCR detection system (Bio-Rad) at $37^{\circ} \mathrm{C}$ in the FAM and/or HEX channels.

Compliance and ethics The author(s) declare that they have no conflict of interest.

Acknowledgements This work was supported by the National Natural Science Foundation of China (31771808 and 32001551), the China Postdoctoral Science Foundation (2020M680779), and the Agricultural Science and Technology Innovation Program of the Chinese Academy of Agricultural Sciences (S2021ZD03). We would like to thank Prof. Dainping Di from Hebei Academy of Agricultural and Forestry for providing RBSDVcarried samples.

\section{References}

Abudayyeh, O.O., Gootenberg, J.S., Kellner, M.J., and Zhang, F. (2019). Nucleic acid detection of plant genes using CRISPR-Cas13. CRISPR J 2, 165-171.

Abudayyeh, O.O., Gootenberg, J.S., Konermann, S., Joung, J., Slaymaker, I.M., Cox, D.B.T., Shmakov, S., Makarova, K.S., Semenova, E., Minakhin, L., et al. (2016). C2c2 is a single-component programmable RNA-guided RNA-targeting CRISPR effector. Science 353.

Ali, Z., Aman, R., Mahas, A., Rao, G.S., Tehseen, M., Marsic, T., Salunke, R., Subudhi, A.K., Hala, S.M., Hamdan, S.M., et al. (2020). iSCAN: an RT-LAMP-coupled CRISPR-Cas12 module for rapid, sensitive detection of SARS-CoV-2. Virus Res 288, 198129.

Bai, G., and Shaner, G. (2004). Management and resistance in wheat and barley to Fusarium head blight. Annu Rev Phytopathol 42, 135-161.

Bluhm, B.H., Flaherty, J.E., Cousin, M.A., and Woloshuk, C.P. (2002). Multiplex polymerase chain reaction assay for the differential detection of trichothecene- and fumonisin-producing species of fusarium in cornmeal. J Food Prot 65, 1955-1961.

Brogan, D.J., Chaverra-Rodriguez, D., Lin, C.P., Smidler, A.L., Yang, T., Alcantara, L.M., Antoshechkin, L., Liu, J., Raban, R.R., Belda-Ferre, P., et al. (2020). A sensitive, rapid, and portable CasRx-based diagnostic assay for SARS-CoV-2. medRxiv doi: 10.1101/2020.10.14.20212795.

Chandra Nayaka, S., Udaya Shankar, A.C., Reddy, M.S., Niranjana, S.R., Prakash, H.S., Shetty, H.S., and Mortensen, C.N. (2009). Control of Fusarium verticillioides, cause of ear rot of maize, by Pseudomonas fluorescens. Pest Manag Sci 65, 769-775.

Chen, J.S., Ma, E., Harrington, L.B., Da Costa, M., Tian, X., Palefsky, J.M., and Doudna, J.A. (2018). CRISPR-Cas12a target binding unleashes indiscriminate single-stranded DNase activity. Science 360, 436-439.

Cheng, Z., Li, S., Gao, R., Sun, F., Liu, W., Zhou, G., Wu, J., Zhou, X., and Zhou, Y. (2013). Distribution and genetic diversity of Southern rice black-streaked dwarf viru s in China. Virol J 10, 307.

Deepa, N., and Sreenivasa, M. (2017). Fusarium verticillioides, a globally important pathogen of agriculture and livestock: a review. J Vet Med Res 4, 1084.

East-Seletsky, A., O’Connell, M.R., Knight, S.C., Burstein, D., Cate, J.H. D., Tjian, R., and Doudna, J.A. (2016). Two distinct RNase activities of CRISPR-C2c2 enable guide-RNA processing and RNA detection. Nature 538, 270-273.

Fang, Y., and Ramasamy, R.P. (2015). Current and prospective methods for plant disease detection. Biosensors 5, 537-561.

Gootenberg, J.S., Abudayyeh, O.O., Lee, J.W., Essletzbichler, P., Dy, A.J., Joung, J., Verdine, V., Donghia, N., Daringer, N.M., Freije, C.A., et al. (2017). Nucleic acid detection with CRISPR-Cas13a/C2c2. Science $356,438-442$.

Gootenberg, J.S., Abudayyeh, O.O., Kellner, M.J., Joung, J., Collins, J.J., and Zhang, F. (2018). Multiplexed and portable nucleic acid detection platform with Cas13, Cas12a, and Csm6. Science 360, 439-444.

Harrington, L.B., Burstein, D., Chen, J.S., Paez-Espino, D., Ma, E., Witte, I.P., Cofsky, J.C., Kyrpides, N.C., Banfield, J.F., and Doudna, J.A. (2018). Programmed DNA destruction by miniature CRISPR-Cas 14 enzymes. Science 362, 839-842.

Jiao, J., Kong, K., Han, J., Song, S., Bai, T., Song, C., Wang, M., Yan, Z., Zhang, H., Zhang, R., et al. (2021). Field detection of multiple RNA viruses/viroids in apple using a CRISPR/Cas12a-based visual assay. Plant Biotechnol J 19, 394-405.

Kellner, M.J., Koob, J.G., Gootenberg, J.S., Abudayyeh, O.O., and Zhang, F. (2019). SHERLOCK: nucleic acid detection with CRISPR nucleases. Nat Protoc 14, 2986-3012.

Konermann, S., Lotfy, P., Brideau, N.J., Oki, J., Shokhirev, M.N., and Hsu, P.D. (2018). Transcriptome engineering with RNA-targeting type VI-D CRISPR effectors. Cell 173, 665-676.e14.

Kushawah, G., Hernandez-Huertas, L., Abugattas-Nuñez Del Prado, J., Martinez-Morales, J.R., DeVore, M.L., Hassan, H., Moreno-Sanchez, I., Tomas-Gallardo, L., Diaz-Moscoso, A., Monges, D.E., et al. (2020). CRISPR-Cas13d induces efficient mRNA knockdown in animal embryos. Dev Cell 54, 805-817.e7.

Liu, T.Y., Knott, G.J., Smock, D.C.J., Desmarais, J.J., Son, S., Bhuiya, A., Jakhanwal, S., Prywes, N., Agrawal, S., Díaz de León Derby, M., et al. (2021). Accelerated RNA detection using tandem CRISPR nucleases. Nat Chem Biol 17, 982-988.

Mahas, A., Aman, R., and Mahfouz, M. (2019). CRISPR-Cas13d mediates robust RNA virus interference in plants. Genome Biol 20, 1-6.

Mulè, G., Susca, A., Stea, G., and Moretti, A. (2004). A species-specific PCR assay based on the calmodulin partial gene for identification of Fusarium verticillioides, $F$. proliferatum and $F$. subglutinans. Eur J Plant Pathol 110, 495-502.

Myhrvold, C., Freije, C.A., Gootenberg, J.S., Abudayyeh, O.O., Metsky, H. C., Durbin, A.F., Kellner, M.J., Tan, A.L., Paul, L.M., Parham, L.A., et al. (2018). Field-deployable viral diagnostics using CRISPR-Cas13. Science 360, 444-448.

Nimse, S.B., Sonawane, M.D., Song, K.S., and Kim, T. (2016). Biomarker detection technologies and future directions. Analyst 141, 740-755.

Qiao, X., Gao, Y., Li, J., Wang, Z., Qiao, H., and Qi, H. (2021). Sensitive analysis of single nucleotide variation by Cas13d orthologs, EsCas13d and RspCas13d. Biotechnol Bioeng 118, 3037-3045.

Rees, H.A., Komor, A.C., Yeh, W.H., Caetano-Lopes, J., Warman, M., Edge, A.S.B., and Liu, D.R. (2017). Improving the DNA specificity and applicability of base editing through protein engineering and protein delivery. Nat Commun 8,1 .

Teng, F., Guo, L., Cui, T., Wang, X.G., Xu, K., Gao, Q., Zhou, Q., and Li, W. (2019). CDetection: CRISPR-Cas12b-based DNA detection with sub-attomolar sensitivity and single-base specificity. Genome Biol 20, 132.

Wang, Z.H., Fang, S.G., Xu, J.L., Sun, L.Y., Li, D.W., and Yu, J.L. (2003). Sequence analysis of the complete genome of rice black-streaked dwarf virus isolated from maize with rough dwarf disease. Virus Genes 27, $163-168$.

Wen, J., Shen, Y., Xing, Y., Wang, Z., Han, S., Li, S., Yang, C., Hao, D., and Zhang, Y. (2020). QTL mapping of resistance to Gibberella ear rot 
in maize. Mol Breeding 40, 94.

Xiong, D., Dai, W., Gong, J., Li, G., Liu, N., Wu, W., Pan, J., Chen, C., Jiao, Y., Deng, H., et al. (2020). Rapid detection of SARS-CoV-2 with CRISPR-Cas12a. PLoS Biol 18, e3000978.

Yan, W.X., Chong, S., Zhang, H., Makarova, K.S., Koonin, E.V., Cheng, D. R., and Scott, D.A. (2018). Cas13d is a compact RNA-targeting type VI CRISPR effector positively modulated by a WYL-domain-containing accessory protein. Mol Cell 70, 327-339.e5.

Yang, Q., Yin, G., Guo, Y., Zhang, D., Chen, S., and Xu, M. (2010). A major QTL for resistance to Gibberella stalk rot in maize. Theor Appl Genet 121, 673-687.

Zetsche, B., Gootenberg, J.S., Abudayyeh, O.O., Slaymaker, I.M., Makarova, K.S., Essletzbichler, P., Volz, S.E., Joung, J., van der Oost, J., Regev, A., et al. (2015). Cpf1 is a single RNA-guided endonuclease of a class 2 CRISPR-Cas system. Cell 163, 759-771.

Zhang, Y., Nguyen, T.M., Zhang, X.O., Wang, L., Phan, T., Clohessy, J.G., and Pandolfi, P.P. (2021). Optimized RNA-targeting CRISPR/Cas13d technology outperforms shRNA in identifying functional circRNAs. Genome Biol 22, 41.

Zhou, D., Wang, X., Chen, G., Sun, S., Yang, Y., Zhu, Z., and Duan, C. (2018). The major fusarium species causing maize ear and Kernel rot and their toxigenicity in Chongqing, China. Toxins 10, 90.

Zhou, H., Su, J., Hu, X., Zhou, C., Li, H., Chen, Z., Xiao, Q., Wang, B., $\mathrm{Wu}, \mathrm{W}$. , Sun, Y., et al. (2020). Glia-to-neuron conversion by CRISPRCasRx alleviates symptoms of neurological disease in mice. Cell 181, 590-603.e16.

Zhou, Y., Xu, Z., Duan, C., Chen, Y., Meng, Q., Wu, J., Hao, Z., Wang, Z., Li, M., Yong, H., et al. (2016). Dual transcriptome analysis reveals insights into the response to Rice black-streaked dwarf virus in maize. J Exp Bot 67, 4593-4609.

Zhou, Y., Zhang, L., Zhang, X., Zu, H., Di, H., Dong, L., Liu, X., Zeng, X., Weng, J., Wang, Z., et al. (2017). Rice black-streaked dwarf virus genome in China: diversification, phylogeny, and selection. Plant Dis 101, 1588-1596.

\section{SUPPORTING INFORMATION}

The supporting information is available online at https://doi.org/10.1007/s11427-021-2028-x. The supporting materials are published as submitted, without typesetting or editing. The responsibility for scientific accuracy and content remains entirely with the authors. 